\title{
ENCUESTAS EN SALUD: INSTRUMENTOS ESENCIALES EN EL SEGUIMIENTO Y EVALUACIÓN DE LOS PROGRAMAS PRESUPUESTALES
}

\author{
José Enrique Velásquez Hurtado1,a, Rosmery Arletty Rivera Sivirichi²,b
}

\begin{abstract}
RESUMEN
El presente artículo analiza el uso de las encuestas de salud en el seguimiento y la evaluación de los programas presupuestales. Desde sus inicios, el año 2008, en el Perú, el presupuesto por resultados (PpR) ha significado no solo una modificación de la organización del presupuesto del Estado, sino un giro hacia una perspectiva basada en evidencia científica que prioriza los resultados en la población y los bienes y servicios necesarios. Las encuestas nacionales de salud han tenido para ello un rol relevante, pues brindan información poblacional a nivel de productos y resultados, información que, además, ha apoyado los cambios en el ciclo de gestión de las intervenciones del Estado, bajo una estructura en la que la disponibilidad de información constituye el mejor insumo para la toma de decisiones. Por último, presenta las consideraciones que debemos tener en cuenta para el uso adecuado de la información que proviene de encuestas en salud y la complementariedad que requiere de otras fuentes de información, de manera que se facilite el abordaje integral en el seguimiento y la evaluación de la cadena de resultados de los programas presupuestales en salud. Las encuestas constituyen herramientas importantes para el seguimiento y evaluación de programas presupuestales, su conocimiento detallado permite mejorar su uso en la toma de decisiones.
\end{abstract}

Palabras clave: Presupuestos; Planificación; Evaluación en salud; Encuestas de salud (Fuente: DeCS BIREME).

\section{HEALTH SURVEYS: ESSENTIALS TOOLS IN THE MONITORING AND EVALUATION OF THE BUDGETARY PROGRAMS}

\begin{abstract}
This article analyzes the use of health surveys in the monitoring and evaluation of the Budgetary Programs. From its beginnings in 2008, in Peru, the Budget for Results (PpR) has meant not only a modification of the organization of the State budget, but a shift towards a perspective based on scientific evidence that prioritizes the results in the population and the necessary goods and services. National health surveys have played a relevant role, since they provide population information at the product and results level, information that has also supported the changes in the management cycle of State interventions, under a structure in which the availability of information constitutes the best input for decision making. Finally, it presents the considerations that must be taken into account for the adequate use of the information that comes from health surveys and the complementarity that it requires of other sources of information, so as to facilitate the integral approach in the monitoring and evaluation of the chain of results of budgetary programs in health. Surveys are important tools for monitoring and evaluating budget programs, their detailed knowledge allows them to improve their use in decision-making.
\end{abstract}

Key words: Budgets; Planning; Health evaluation; Health surveys (Source: MeSH NLM).

\section{INTRODUCCIÓN}

La Gestión por Resultados (GpR) surge como necesidad de una mayor eficiencia en la asignación y el uso de los recursos públicos, orientada al logro de resultados y no solo al cumplimiento de funciones y actividades. Basada en el concepto de decisiones informadas, constituye una estrategia de gestión pública que busca una toma de decisiones sobre la base de información confiable acerca de los efectos que la acción gubernamental tiene en la sociedad, considerando para ello distintos elementos del ciclo de la gestión (planificación, presupuesto, gestión financiera, gestión de programas y proyectos, seguimiento y evaluación) ${ }^{(1)}$.

Aunque son claras las ventajas de tener una gestión pública más eficiente, efectiva y abierta, el cómo hacerlo no constituye necesariamente un aspecto tan sencillo de

\footnotetext{
Universidad Peruana Cayetano Heredia. Lima, Perú

Universidad del Pacífico. Lima, Perú

a Médico, magíster en Salud Pública; ${ }^{\text {b }}$ economista, magíster en Gestión Pública

Recibido: 02/08/2017 Aprobado: 20/09/2017 En línea: 29/09/2017
}

Citar como: Velásquez Hurtado JE, Rivera Sivirichi RA. Encuestas en salud: instrumentos esenciales en el seguimiento y evaluación de los programas presupuestales. Rev Peru Med Exp Salud Publica. 2017;34(3):512-20. doi: 10.17843/rpmesp.2017.343.3031 
solucionar. En América Latina y el Caribe, recién desde principios de este siglo la GpR ha sido una preocupación constante, si bien sus inicios datan desde la crisis de los años ochenta. El Banco Interamericano de Desarrollo, en los años 2007 y 2013, comparó el estado y avance de 24 países de América Latina, entre ellos el Perú, encontrando una evolución positiva de las capacidades institucionales para la implementación de la GpR, siendo los más débiles los aspectos relacionados con el presupuesto por resultados $(\mathrm{PpR})$ y el seguimiento y evaluación (2).

En el Perú, desde fines de la década anterior, se ha venido implementando una propuesta de $\mathrm{PpR}$, que estructura el ciclo de presupuesto en base a resultados en la población y los bienes y servicios necesarios para lograrlos, acompañado de acciones de rendición de cuentas en las que los responsables o gerentes dan cuenta de los productos entregados y resultados logrados. Lo primero presupone la definición previa de resultados y productos (bienes y servicios), y los costos que requieren para su implementación, lo segundo un cambio significativo en la disponibilidad de la información, el manejo de la misma por los tomadores de decisiones y un cambio de mentalidad y transparencia.

La definición de resultados y productos requiere de la identificación de la teoría de cambio de las intervenciones del Estado y, si bien puede presentarse de múltiples formas (p.e. modelos lógicos, marcos lógicos, cadenas de resultados, u otra), estas representan, finalmente, la cadena causal que brinda la definición lógica y plausible de cómo una secuencia de insumos, actividades y productos relacionados directamente con la intervención interactúan y establecen las vías por las que se logran los resultados ${ }^{(3)}$ (Figura 1).
La definición de la teoría de cambio permite identificar los indicadores para cada uno de los aspectos de la secuencia, así como la relación entre ellos, permitiendo establecer los aspectos cruciales para el seguimiento, en el primer caso, y para la evaluación, en el segundo. El seguimiento, entonces, se realiza en base a los indicadores que provienen de la cadena de causalidad que conecta insumo con el resultado final, y la evaluación, si se realiza durante la planificación, se centra en el diseño del programa o el diseño del modelo de intervención, si es durante la implementación del programa se centra en la eficiencia en la entrega del producto o la efectividad en el logro de los resultados, y si es durante el mantenimiento, en la efectividad.

En el marco del PpR, las actividades principales de seguimiento comprenden: 1) Seguimiento de la ejecución financiera y física; 2) Seguimiento de indicadores de desempeño, y 3) Seguimiento del gasto social; y las de evaluación comprenden: 1) Evaluaciones de diseño y ejecución presupuestal (EDEP), y 2) Evaluaciones de impacto, rescatando la importancia que estas últimas sean además independientes, es decir, sean realizadas por personas naturales o jurídicas diferentes a las que diseñan y/o ejecutan las acciones que son objeto de la evaluación ${ }^{(4)}$.

En el marco de lo anterior, la información representa una necesidad inmediata y las encuestas nacionales de salud un instrumento valioso, no solo por su periodicidad anual, sino también por su recojo estandarizado de información por una institución independiente y su representatividad poblacional. Pueden proporcionar información sobre el grado de cumplimiento de los productos y resultados, además de contribuir con la verificación de los efectos que tendría la gestión pública en la población.

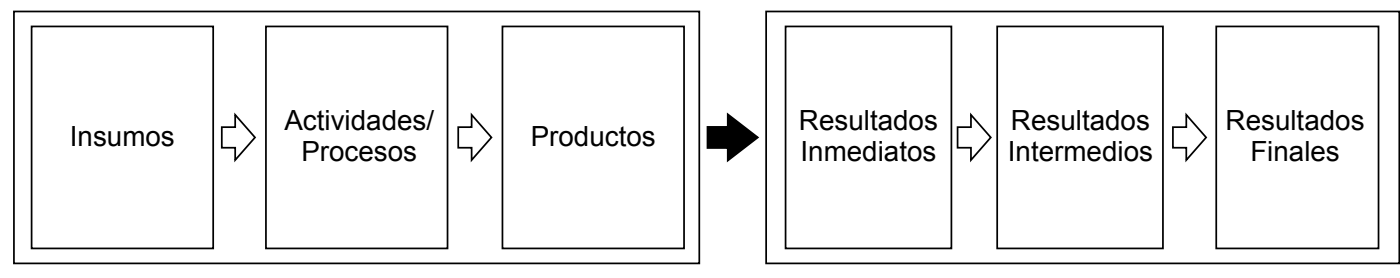

\begin{tabular}{|c|c|c|c|}
\hline $\begin{array}{c}\text { RECURS } \\
\text { invertimos? }\end{array}$ & $\begin{array}{c}\text { ¿Qué } \\
\text { HACEMOS? }\end{array}$ & $\begin{array}{c}\text { ¿Qué } \\
\text { SERVICIOS } \\
\text { producimos? }\end{array}$ & $\begin{array}{c}\text { ¿Qué CAMBIOS se producen en la } \\
\text { POBLACIÓN que recibe los servicios? }\end{array}$ \\
\hline $\begin{array}{c}\text { Programación } \\
\text { y ejecución } \\
\text { presupuestal } \\
\text { Adquisición } \\
\text { de insumos }\end{array}$ & $\begin{array}{c}\text { Actividades } \\
\text { necesarias } \\
\text { para la } \\
\text { producción } \\
\text { de bienes y } \\
\text { servicios }\end{array}$ & $\begin{array}{c}\text { Bienes y } \\
\text { servicios } \\
\text { Producidos }\end{array}$ & $\begin{array}{c}\text { Cambios o beneficios logrados } \\
\text { para los individuos, familias, } \\
\text { grupos u organizaciones }\end{array}$ \\
\hline
\end{tabular}

Figura 1. Cadena de resultados de una intervención 
Los programas presupuestales en salud tienen resultados y productos que pueden ser medidos en encuestas nacionales. Si bien la encuesta más conocida y utilizada es la Encuesta Nacional Demográfica y de Salud Familiar (ENDES) ${ }^{(5)}$ existen otras encuestas como la Encuesta Nacional de Hogares (ENAHO) ${ }^{(6)}$, la Encuesta Nacional de Programas Presupuestales (ENAPRES) ${ }^{(7)}$ o algunas más específicas como la Encuesta a Establecimientos de Salud en la Atención del Control de Crecimiento, Desarrollo y Vacunas en Menores de un año (ENCRED) ${ }^{(8)}$, la Encuesta Nacional Especializada sobre Discapacitados (ENEDIS) ${ }^{(9)}$, la Encuesta a Establecimientos de Salud con Funciones Obstétricas y Neonatales (ENESA) ${ }^{(10)}$, o la Encuesta de Riesgo Anual de Infección por Tuberculosis (RAIT) ${ }^{(11)}$.

\section{PROGRAMAS PRESUPUESTALES EN SALUD}

La salud constituye un derecho y un bien público que, según la Constitución Política Nacional debe ser velada en su conservación y restablecimiento por todos (Estado y las personas). En este contexto, la protección que requiere la salud de las personas obliga al Estado al empleo de herramientas que garanticen el uso eficiente y con criterios de equidad y calidad de sus principales prestaciones. El presupuesto público y el $\mathrm{PpR}$ cumplen un papel fundamental en las funciones del Estado, de asignación, distribución y estabilización (12).

La implementación del PpR requiere de programas presupuestales, que constituyen las unidades de programación con las que las instituciones públicas realizan la programación de acciones integradas y articuladas, relacionadas causalmente con los resultados de interés en la población y las políticas públicas. Si bien, el Gobierno Peruano a través de la aprobación de la Ley 28927-Ley de Presupuesto del Sector Público para el año Fiscal 2007, incorpora los elementos básicos del PpR a finales del año 2007, no es hasta el año 2008 en que se desarrolla la metodología de lo que constituyó la programación presupuestal estratégica, basada en once actividades prioritarias definidas en la Ley, de las cuales seis eran relacionadas con la salud de la madre y el niño ${ }^{(13)}$.

Aunque en la teoría, el PpR aparece como una alternativa idónea para asignar los recursos públicos, en la práctica requiere de tareas bastante complejas, revisión sistemática de la evidencia, definición operacional de los modelos de gestión de las intervenciones, además de trabajo intersectoriales e intergubernamentales, de manera que desde el diseño se identifiquen indicadores relacionados con su seguimiento. Por ello, durante el año 2008 se definieron solo cinco programas presupuestales, dos de los cuales se relacionaban con la salud de la madre y los niños, uno con la identificación de los individuos, uno con la educación y uno con el acceso a servicios sociales básicos y oportunidades de mercado. Para el año 2017, el presupuesto nacional considera un total de 90 programas presupuestales, de los cuales 10 están directamente relacionados con el sector Salud (Tabla 1).

EIPpR ha requerido, además, del desarrollo de instrumentos ad-hoc a la realidad peruana y a las restricciones que podemos encontrar en un país heterogéneo, a fin de contar con herramientas que permitan asegurar que la implementación se está realizando según los objetivos planteados. Los instrumentos complementarios más reconocidos son: 1) la programación presupuestaria (actualmente tenemos 90 programas presupuestales); 2) el seguimiento de indicadores de resultados y productos; 3) la realización de evaluaciones independientes (evaluaciones de diseño y ejecución presupuestal EDEP, y las evaluaciones de impacto), y 4) los incentivos a la gestión ${ }^{(14)}$. Para todos se requiere de información que provenga de registros administrativos (como en el seguimiento de indicadores de producto) o de encuestas nacionales, estudios o censos (como para las evaluaciones de impacto).

\section{SEGUIMIENTO Y EVALUACIÓN DE PROGRAMAS PRESUPUESTALES USANDO ENCUESTAS DE SALUD}

En el marco del PpR, el seguimiento se define como un proceso continuo que recopila y analiza información de indicadores de producto y resultado; y la evaluación como un análisis sistemático y objetivo de un programa, sea relacionada a su diseño, gestión, resultados de desempeño e impacto; generando evidencia para mejorar su diseño o implementación. El seguimiento puede corresponder a: 1) la ejecución financiera y física, que se realiza desde el año 2009 con la información del Sistema Integrado de Administración Financiera (SIAF); 2) los indicadores de desempeño, que utilizan las encuestas realizadas por el Instituto Nacional de Estadística e Informática (INEI) y registros administrativos de las entidades del Estado, y 3) el gasto social, que utiliza también la información del SIAF. Por su parte, la evaluación puede corresponder a: 1) evaluaciones de diseño y ejecución presupuestal (EDEP), orientada al diseño como a la implementación y desempeño en la entrega de los bienes y servicios, y 2) evaluaciones de impacto, que permiten estimar el logro atribuible al programa en el resultado alcanzado.

Ambos, seguimiento y evaluación, pueden requerir de encuestas para su información. Si bien, las encuestas como ENDES o ENAHO existen desde antes de la generación de los programas presupuestales, estas encuestas han sido diseñadas para responder a los principales indicadores de salud del sector, al igual que los programas presupuestales 
en salud. Los principales usuarios de las encuestas son las autoridades a nivel nacional, regional y local, así como investigadores acerca de los principales temáticas de salud en el país, desde su inicio las encuestas han sufrido variaciones en las secciones que estructuran las encuestas y anualmente se revisa la estructura de preguntas en cada sección, lo que abre la posibilidad al sector de proponer la incorporación o retiro de algunas variables, con la finalidad de asegurar que la estimación de los principales indicadores de salud a nivel de resultado y productos se recojan de manera periódica.
Actualmente, el INEI a través de la aplicación de encuestas apoya con el seguimiento de 335 indicadores, correspondientes a 60 programas presupuestales ${ }^{(15,16)}$, de los cuales 82 indicadores corresponden a los 10 programas presupuestales del sector Salud (17 indicadores de resultado para 5 programas presupuestales y 65 indicadores de producto para 9 programas presupuestales). La Tabla 1 muestra las encuestas que son utilizadas actualmente para el seguimiento de los indicadores de resultado y producto, la mayor cantidad proviene de la ENDES y la ENAPRES, de las cuales es posible obtener información anual (al igual

Tabla 1. Programas presupuestales, encuestas utilizadas para seguimiento y evaluaciones identificadas del sector Salud

\begin{tabular}{|c|c|c|}
\hline Programa presupuestal & $\begin{array}{l}\text { Indicadores y encuestas } \\
\text { utilizadas en el seguimiento }\end{array}$ & Evaluaciones identificadas \\
\hline $\begin{array}{l}\text { Programa presupuestal } \\
\text { 0001. Programa Articulado } \\
\text { Nutricional }\end{array}$ & $\begin{array}{c}4 \text { indicadores de resultado } \\
\text { (ENDES), } 12 \text { indicadores de } \\
\text { producto ( } 11 \text { de ENDES, } 1 \text { de } \\
\text { ENAHO) }\end{array}$ & $\begin{array}{l}3 \text { evaluaciones de diseño y ejecución de presupuesto } \\
\text { (1 servicios de vacunación, ENDES y MONIN; } 1 \text { de atención de } \\
\text { enfermedades diarreicas-EDA y las infecciones respiratorias } \\
\text { agudas-IRA, ENDES; } 1 \text { CRED y Municipios Saludables, } \\
\text { ENDES, ENCRED y ENAHO); } 2 \text { evaluaciones de impacto } \\
\text { (1 evaluación de impacto del PAN sobre la DCI en niños } \\
\text { menores de } 5 \text { años, ENDES; } 1 \text { evaluación de resultados del } \\
\text { PAN, ENDES) }\end{array}$ \\
\hline $\begin{array}{l}\text { Programa presupuestal } 0002 . \\
\text { Salud Materno Neonatal }\end{array}$ & $\begin{array}{c}8 \text { indicadores de resultado } \\
\text { (4 de ENDES y } 4 \text { de ENESA), } 7 \\
\text { indicadores de producto (ENDES) }\end{array}$ & $\begin{array}{l}1 \text { evaluación del diseño y ejecución de presupuesto } \\
\text { (1 evaluación de la atención del parto normal, ENDES y ENESA) }\end{array}$ \\
\hline $\begin{array}{l}\text { Programa presupuestal } 0016 . \\
\text { TBC/VIH-SIDA }\end{array}$ & $\begin{array}{l}4 \text { indicadores de producto } \\
\text { (ENDES) }\end{array}$ & $\begin{array}{l}1 \text { evaluación del diseño y ejecución de presupuesto ( } 1 \\
\text { evaluación del componente tuberculosis del PP TB-VIH, } \\
\text { ENDES y RAIT) }\end{array}$ \\
\hline $\begin{array}{l}\text { Programa presupuestal } 0017 . \\
\text { Enfermedades Metaxénicas y } \\
\text { Zoonosis }\end{array}$ & $\begin{array}{l}8 \text { indicadores de producto } \\
\text { (ENAPRES) }\end{array}$ & No hay evaluaciones identificadas \\
\hline $\begin{array}{l}\text { Programa presupuestal } \\
\text { 0018. Enfermedades No } \\
\text { Transmisibles }\end{array}$ & $\begin{array}{l}3 \text { indicadores de resultado } \\
\text { (ENDES), } 21 \text { indicadores de } \\
\text { producto (ENDES) }\end{array}$ & No hay evaluaciones identificadas \\
\hline $\begin{array}{l}\text { Programa presupuestal } 0024 . \\
\text { Prevención y Control del } \\
\text { Cáncer }\end{array}$ & $\begin{array}{l}8 \text { indicadores de producto } \\
\text { (ENDES) }\end{array}$ & $\begin{array}{l}1 \text { evaluación del diseño y ejecución de presupuesto } \\
\text { (1 evaluación EDEP del PP, ENDES) }\end{array}$ \\
\hline $\begin{array}{l}\text { Programa presupuestal } 0068 . \\
\text { Reducción de Vulnerabilidad } \\
\text { y Atención de Emergencias } \\
\text { por Desastres }\end{array}$ & $\begin{array}{l}3 \text { indicadores de resultado } \\
\text { (ENAPRES) }\end{array}$ & No hay evaluaciones identificadas \\
\hline $\begin{array}{l}\text { Programa presupuestal } 0104 . \\
\text { Reducción de la Mortalidad } \\
\text { por Emergencias y Urgencias } \\
\text { Médicas }\end{array}$ & $\begin{array}{l}2 \text { indicadores de resultado } \\
\text { (ENAPRES) }\end{array}$ & $\begin{array}{l}1 \text { evaluación del diseño y ejecución de presupuesto } \\
\text { (1 evaluación EDEP del PP, ENAHO y ENDES) }\end{array}$ \\
\hline $\begin{array}{l}\text { Programa presupuestal } \\
\text { 0129. Prevención y Manejo } \\
\text { de Condiciones Secundarias } \\
\text { de Salud en Personas con } \\
\text { Discapacidad }\end{array}$ & $\begin{array}{l}\text { No cuenta con una encuesta para } \\
\text { seguimiento }\end{array}$ & No hay evaluaciones identificadas \\
\hline $\begin{array}{l}\text { Programa presupuestal } 0131 . \\
\text { Control y Prevención en } \\
\text { Salud Mental }\end{array}$ & $\begin{array}{c}2 \text { indicadores de producto } \\
\text { (ENDES) }\end{array}$ & No hay evaluaciones identificadas \\
\hline
\end{tabular}

El año fiscal 2017 cuenta con un total de 90 programa presupuestales (PP), la organización por funciones, permite identificar en la función salud 12 PP, no todos los PP de la función salud corresponden al sector Salud, el cual contempla 10 PP

ENDES: Encuesta Nacional Demográfica y de Salud Familiar, ENAHO: Encuesta Nacional de Hogares, ENAPRES: la Encuesta Nacional de Programas Presupuestales, ENCRED: Encuesta a Establecimientos de Salud en la Atención del Control de Crecimiento, Desarrollo y Vacunas en Menores de un año, ENESA: Encuesta a Establecimientos de Salud con Funciones Obstétricas y Neonatales, RAIT: Encuesta de Riesgo Anual de Infección por Tuberculosis, MONIN: Monitoreo Nacional de Indicadores Nutricionales, PAN: Programa Articulado Nutricional, DCI: Desnutrición Crónica Infantil, EDEP: Evaluaciones de Diseño y Ejecución Presupuestal

Fuente: Ministerio de Salud, Ministerio de Economía y Finanzas 
que de la ENAHO, la ENESA dispone de información anual o cada dos años).

Las encuestas permiten obtener información periódica (generalmente anual) a nivel nacional, por ámbitos urbano-rural, departamentos y regiones naturales. Aunque es usual utilizar solo el valor puntual de los estimados, debe tenerse en cuenta que la información proviene de una muestra por lo que está sujeta a un error estadístico variable. Los indicadores de los programas presupuestales que proceden de encuestas pueden ser verificados a través de los reportes de las encuestas de donde provienen (p.e. reportes de la ENDES o ENAHO), reportes específicos de PpR (p.e. el INEI los publica anualmente en un libro presente en su web institucional) o vía el aplicativo RESULTA de la web del Ministerio de Economía y Finanzas (MEF) (http://apps5.mineco.gob. pe/resulta).

En caso de las evaluaciones, cinco de los programas presupuestales de salud cuentan con una EDEP o una evaluación de impacto, es posible que al momento de la publicación de este artículo haya otro en proceso; con la finalidad de facilitar en análisis y el contraste de la información que contiene y el uso de encuestas solo se han tomado aquellas publicadas o cuyo informe se encuentra ubicable. Así, para los programas presupuestales que cuentan con una evaluación, el programa presupuestal que cuenta con mayor cantidad de evaluaciones es el PAN, hay un total de ocho evaluaciones de las cuales una es evaluación de impacto, el resto corresponden a EDEP. La ENDES y la ENAHO fueron las dos encuestas más utilizadas (Tabla 1).

Las encuestas tienen ventajas que pueden ser utilizadas a favor del seguimiento o la evaluación de los programas presupuestales; sin embargo, debemos reconocer que también tienen limitaciones, no permiten obtener datos para poblaciones menores a una región, como distritos, para algunas poblaciones vulnerables como la población indígena, no brindan información sobre el diseño de los programas o sobre la implementación de las intervenciones, aunque sí permiten la caracterización del problema y tienen una extensión determinada que, en caso de extenderse, podría afectar la calidad de la información.

Si bien hay información que puede ser consultada para cada uno de los programas presupuestales de salud, hemos decidido profundizar más sobre los programas articulado nutricional (PAN) y salud materno neonatal (SMN), fueron los dos primeros programas presupuestales en salud y se concentran en problemas históricamente priorizados por su impacto en la población, ello ha representado que desde su formulación se disponga de una mayor evidencia científica y naturalmente de mayor discusión, así como de la representatividad de sus indicadores en encuestas nacionales como la ENDES.

\section{EL USO DE ENCUESTAS PARA EL SEGUIMIENTO DE LOS PROGRAMAS PRESUPUESTALES RELACIONADOS CON LA SALUD MATERNO INFANTIL}

Los programas PAN y SMN buscan mejorar la salud materno infantil, el primero está orientado a la reducción de la desnutrición crónica en los niños menores de cinco años y el segundo a la reducción de la morbimortalidad materna y neonatal; ambos priorizan y articulan un conjunto de intervenciones de carácter intersectorial e intergubernamental buscando generar sinergias entre diferentes sectores e intervenciones efectivas que permitan asegurar el logro de los resultados previstos.

La mayor parte de los indicadores de desempeño proviene de la ENDES, que asegura la disponibilidad de información de manera periódica (anual para las regiones y semestral para el país), algo similar ocurre con la ENAHO que permite contar con información organizada de manera trimestral; la excepción lo constituye la ENESA que se recoge cada uno o dos años ${ }^{(17)}$. Adicionalmente, el INEI publica de manera anual el resultado de sus encuestas relacionadas con los programas presupuestales ${ }^{(18)}$.

EI PAN tiene como indicador de resultado final la prevalencia de desnutrición crónica en menores de 5 años (patrón OMS), indicador que ha mostrado una tendencia decreciente sostenida; el programa SMN tiene dos resultados finales: la tasa de mortalidad materna por 100 mil nacidos vivos y la tasa de mortalidad neonatal, ambos también con una tendencia decreciente, aunque el primero de estos indicadores no se obtiene anualmente (el último valor procedente de la ENDES es de 93 por 100 mil nacidos vivos y corresponde al período 2004-2010) ${ }^{(19)}$.

El análisis de estos indicadores necesita algunas consideraciones previas: 1) debe ser realizado en conjunto con el resto de indicadores que conforman su cadena de valor; 2) reconocer las limitaciones que tienen las encuestas para mediciones poco frecuentes y que necesitarían tamaños de muestra mayores, y 3 ) la representatividad para los indicadores de programas presupuestales llegará hasta regiones (en la mayoría de casos), tener niveles mayores de desagregación (provincias, distritos u otros) necesitará de otras fuentes de información.

El seguimiento de ambos programas ha permitido verificar la tendencia de los indicadores de resultado (finales, intermedios e inmediatos) a nivel nacional (Tabla 2). La desagregación por ámbito urbano y rural; costa, sierra y selva, y por regiones, incluyendo Lima Metropolitana ${ }^{(20)}$ permite analizar brechas en algunos 
Tabla 2. Tendencia de los indicadores del programa presupuestal 001. Programa Articulado Nutricional (PAN) y del programa presupuestal 002. Salud Materno Neonatal (SMN)

\begin{tabular}{|c|c|c|c|c|c|c|c|c|c|}
\hline \multirow{2}{*}{ Indicadores } & \multicolumn{9}{|c|}{ Años } \\
\hline & 2008 & 2009 & 2010 & 2011 & 2012 & 2013 & 2014 & 2015 & 2016 \\
\hline \multicolumn{10}{|l|}{ Programa presupuestal 001. Programa Articulado Nutricional } \\
\hline \multicolumn{10}{|l|}{ Resultado final } \\
\hline $\begin{array}{l}\text { Prevalencia de desnutrición crónica en menores de } 5 \text { años } \\
\text { (patrón OMS) }\end{array}$ & 27,5 & 23,8 & 23,2 & 19,5 & 18,1 & 17,5 & 14,6 & 14,4 & 13,1 \\
\hline \multicolumn{10}{|l|}{ Resultado Intermedio } \\
\hline Prevalencia de anemia en menores de 36 meses & 57,8 & 50,4 & 50,3 & 41,6 & 44,5 & 46,4 & 46,8 & 43,5 & 43,6 \\
\hline $\begin{array}{l}\text { Proporción de menores de } 36 \text { meses con lactancia exclusiva hasta } \\
\text { los } 6 \text { meses }\end{array}$ & 65,7 & 68,5 & 68,3 & 70,6 & 67,6 & 72,3 & 68,4 & 65,2 & 69,8 \\
\hline Incidencia de enfermedad diarreica aguda en menores de 36 meses & 17,9 & 18,0 & 18,9 & 17,6 & 15,3 & 13,7 & 15,3 & 15,5 & 15,0 \\
\hline Incidencia de infección respiratoria aguda en menores de 36 meses & 19,9 & 17,2 & 18,3 & 17,8 & 14,6 & 14,8 & 17,5 & 16,3 & 16,9 \\
\hline Incidencia de bajo peso al nacer $(<2,5 \mathrm{Kg})$ & 7,2 & 7,1 & 8,0 & 7,1 & 7,4 & 7,8 & 6,4 & 7,5 & 7,0 \\
\hline \multicolumn{10}{|l|}{ Resultado inmediato } \\
\hline Porcentaje de hogares con acceso al abastecimiento de agua segura & 30,1 & 27,6 & 25,8 & 24,4 & 25,2 & 28,0 & 28,4 & 28,2 & 31,3 \\
\hline Proporción de hogares con agua tratada & ND & 91,1 & 91,5 & 93,3 & 93,9 & 95,0 & 95,6 & 95,7 & 96,1 \\
\hline $\begin{array}{l}\text { Proporción de menores de } 12 \text { meses con vacunas básicas } \\
\text { completas para su edad }\end{array}$ & ND & 60,4 & 66,5 & 77,2 & 76,8 & 63,2 & 55,7 & 69,4 & 74,0 \\
\hline $\begin{array}{l}\text { Proporción de menores de } 36 \text { meses con Controles de Crecimiento y } \\
\text { Desarrollo completos de acuerdo a su edad }\end{array}$ & 21,6 & 27,7 & 40,0 & 47,3 & 50,8 & 50,5 & 52,4 & 54,9 & 58,2 \\
\hline $\begin{array}{l}\text { Proporción de menores de } 36 \text { meses con vacunas básicas } \\
\text { completas para su edad }\end{array}$ & ND & 53,7 & 60.8 & 71,5 & 72,8 & 66,1 & 61,1 & 69,4 & 71,4 \\
\hline $\begin{array}{l}\text { Proporción de menores de } 36 \text { meses que recibieron suplemento de } \\
\text { hierro }\end{array}$ & 13,1 & 14,1 & 18,4 & 17,0 & 19,6 & 23,6 & 24,5 & 30,3 & 29,2 \\
\hline $\begin{array}{l}\text { Proporción de niños de } 1 \text { mes a menos de } 12 \text { meses con vacunas } \\
\text { de neumococo completas para su edad }\end{array}$ & ND & 29,0 & 57,8 & 74,4 & 76,6 & 79,2 & 79,5 & 82,2 & 82,9 \\
\hline $\begin{array}{l}\text { Proporción de niños de } 1 \text { mes a menos de } 12 \text { meses con vacunas } \\
\text { de rotavirus completas para su edad }\end{array}$ & ND & 36,5 & 60,5 & 77,5 & 81,1 & 78,1 & 78,2 & 80,9 & 81,3 \\
\hline $\begin{array}{l}\text { Proporción de niños y niñas con vacunas completas de acuerdo a } \\
\text { su edad }\end{array}$ & 20,6 & 25,1 & 22,3 & 27,8 & 27,0 & 23,1 & 20,6 & 23,6 & 24,5 \\
\hline Proporción de gestantes que reciben suplemento de hierro & 79,0 & 80,1 & 86,1 & 87,8 & 88,5 & 89,9 & 88,9 & 91,1 & 93,1 \\
\hline \multicolumn{10}{|l|}{ Programa presupuestal 002 . Salud Materno Neonatal } \\
\hline \multicolumn{10}{|l|}{ Resultado final } \\
\hline Tasa de mortalidad neonatal & 13,0 & 12,8 & 11,1 & 9,8 & 12,6 & 12,0 & 11,5 & 10,7 & ND \\
\hline \multicolumn{10}{|l|}{ Resultado intermedio } \\
\hline Tasa global de fecundidad & 2,5 & 2,6 & 2,5 & 2,6 & 2,6 & 2,4 & 2,5 & 2,5 & 2,5 \\
\hline $\begin{array}{l}\text { Proporción de mujeres en unión que usa algún método moderno de } \\
\text { planificación familiar }\end{array}$ & 49,2 & 50,0 & 50,5 & 51,1 & 51,8 & 51,3 & 52,2 & 53,3 & 54,3 \\
\hline Cobertura de parto institucional & 80 & 81,3 & 83,2 & 83,8 & 85,9 & 88 & 89,3 & 90,7 & 91,9 \\
\hline $\begin{array}{l}\text { Proporción de partos atendidos por personal de salud en } \\
\text { establecimientos de salud }\end{array}$ & ND & 63,7 & 67,3 & 67,4 & 70 & ND & ND & ND & ND \\
\hline $\begin{array}{l}\text { Proporción de recién nacidos vivos menores de } 37 \text { semanas de } \\
\text { gestación }\end{array}$ & 10,4 & 15 & 16,2 & 15,8 & 17,5 & 18,5 & 17,2 & 19 & 21,1 \\
\hline \multicolumn{10}{|l|}{ Resultado Inmediato } \\
\hline $\begin{array}{l}\text { Proporción de mujeres con demanda no satisfecha de métodos de } \\
\text { planificación familiar }\end{array}$ & 8,6 & 7,2 & 6,9 & 6,1 & 8,2 & 9 & 8,6 & 6,5 & 6 \\
\hline $\begin{array}{l}\text { Proporción de EESS calificados como FONB en establecimientos } \\
\text { estratégicos }\end{array}$ & ND & ND & ND & 0,011 & 0,028 & 0,000 & ND & 0,000 & ND \\
\hline $\begin{array}{l}\text { Proporción de EESS calificados como FONE I en establecimientos } \\
\text { estratégicos }\end{array}$ & ND & ND & ND & 0,000 & 0,000 & 0,000 & ND & 0,000 & ND \\
\hline $\begin{array}{l}\text { Proporción de EESS calificados como FONE II en establecimientos } \\
\text { estratégicos }\end{array}$ & ND & ND & ND & 0,000 & 0,000 & 0,000 & ND & 0,000 & ND \\
\hline $\begin{array}{l}\text { Proporción de gestantes y puérperas con complicaciones atendidas } \\
\text { en cuidados intensivos generales para adultos de los EESS que } \\
\text { cumplen FONE II con la condición de egreso "sano" }\end{array}$ & ND & ND & ND & ND & ND & 0,9 & ND & ND & ND \\
\hline $\begin{array}{l}\text { Proporción de gestantes y puérperas con complicaciones atendidas } \\
\text { en cuidados intensivos generales para adultos de los EESS que } \\
\text { cumplen FONE I con la condición de egreso "sano" }\end{array}$ & ND & ND & ND & ND & ND & 0,8 & ND & ND & ND \\
\hline $\begin{array}{l}\text { Proporción de partos atendidos en EESS calificados para cumplir } \\
\text { FONB en establecimientos estratégicos }\end{array}$ & ND & ND & ND & ND & ND & 0,4 & ND & ND & ND \\
\hline Proporción de gestantes con 6 atenciones prenatales & 80 & 80,8 & 81 & 83,5 & 84,5 & 87,3 & 86,6 & 88,4 & 88,9 \\
\hline $\begin{array}{l}\text { Proporción de gestantes con por lo menos un control prenatal en el } \\
\text { primer trimestre de gestación }\end{array}$ & 72,2 & 72,4 & 73 & 74,9 & 75 & 77,4 & 77,3 & 79,8 & 80,2 \\
\hline $\begin{array}{l}\text { Proporción de mujeres en edad fértil que conoce algún método de } \\
\text { planificación familiar }\end{array}$ & 99,3 & 99,3 & 99,6 & 99,5 & 99,6 & 99,8 & 99,7 & 99,8 & ND \\
\hline
\end{tabular}

ND: No hay datos, FONB: Establecimiento con funciones obstétricas y neonatales básicas, FONE: Establecimiento con funciones obstétricas $y$ neonatales esenciales, EESS: Establecimiento de Salud

Fuente: Instituto Nacional de Estadística e Informática, Ministerio de Economía y Finanzas 
ámbitos que no puede ser visualizados por ser el nacional un promedio. Ello permite cumplir parcialmente con la primera de las consideraciones anteriormente descritas, pues permite realizar la modificación de los resultados relacionados entre sí, ello permite en caso del PAN no solo verificar la tendencia decreciente de la desnutrición crónica y contrastar además las cifras con metas establecidas en los diferentes niveles de desagregación, sino también mostrar que algunos aspectos como la anemia en menores de 36 meses, lactancia materna exclusiva, la incidencia de infecciones respiratorias agudas (IRA), enfermedades diarreicas agudas (EDA) o bajo peso al nacer, tienen una tendencia casi horizontal en el nivel nacional. Conocer la situación de los indicadores ha permitido implementar paquetes de intervención intersectorial en función a la situación de los principales indicadores de salud, así como poner en la agenda pública la priorización de los recursos para la implementación de estos paquetes de intervenciones efectivas basadas en evidencia, basados en la cadena de valor o ruta crítica que asegura el logro de los resultados.

Algo similar se puede observar con el programa SMN, se puede observar una disminución del indicador de resultado, con mejora de algunos indicadores como el acceso de métodos de planificación familiar, la cobertura de parto institucional o el acceso temprano al control prenatal, pero otros sin mayor variación como la tasa global de fecundidad.

\section{EL USO DE ENCUESTAS PARA LA EVALUACIÓN DE LOS PROGRAMAS PRESUPUESTALES RELACIONADOS CON LA SALUD MATERNO INFANTIL}

Aunque las encuestas por sí mismas no permiten generar toda la información que se requiere para una evaluación, sobre todo la evaluación de impacto, es posible utilizarlas para ver asociaciones preliminares que puedan ser analizadas con mayor profundidad a través de estudios experimentales, pseudoexperimentales o longitudinales con líneas de base y seguimiento de la entrega de las intervenciones. Los Programas PAN y SMN cuentan con evaluaciones EDEP que han usado encuestas nacionales, además de una evaluación de impacto realizada para el PAN.

En 2011, a través de una EDEP, el MEF y el Ministerio de Salud (MINSA) evaluaron en el diseño del PAN la inclusión e implementación del servicio de vacunación de la Estrategia Sanitaria Nacional de Inmunizaciones, encontrándola como una intervención clave y reconocida por la evidencia científica como efectiva, aunque detallando algunas limitaciones operacionales que debían ser corregidos en la gestión de la estrategia a fin de responder de manera efectiva a las necesidades del programa (21), la encuesta ENDES proporcionó los datos de vacunación e infecciones (IRA y EDA). El año 2013, la EDEP relacionada con la atención de IRA y EDA mostró también aspectos importantes a mejorar en su implementación, además de resaltar la complementariedad de los datos con aquellos proporcionados por registros administrativos y la necesidad de controlar el efecto de otras intervenciones o factores determinantes, a fin de aislar de mejor manera el efecto esperado de ambos problemas sobre el resultado final. La información de los resultados fue obtenida de la ENDES, pero complementada con información administrativa de las atenciones de salud, programación presupuestal, entre otras ${ }^{(22)}$.

Las EDEP para atención del parto normal (23) y del crecimiento y desarrollo (CRED) y municipios saludables ${ }^{(24)}$, realizadas los años 2013 y 2015 respectivamente, utilizaron, además de la ENDES, otras encuestas como la ENESA para ver capacidad resolutiva para la atención de parto, y la ENCRED y ENAHO para el servicio CRED y su acceso por niveles de pobreza. La información, igualmente, fue complementada para ver indicadores de procesos, insumos y programación presupuestal con registros administrativos que facilitaron la información de ejecución presupuestal.

EI PAN cuenta, además, con una evaluación de impacto sobre la desnutrición crónica en niños menores de cinco años ${ }^{(25)}$, que utilizó la ENDES para, a través de dos técnicas no experimentales, cohortes de nacimiento y controles a nivel de regiones, verificar que a partir del año 2008 , las cohortes de nacimiento posterior disminuyeron la probabilidad de ser desnutrido en aproximadamente un $5 \%$ y que el efecto promedio del PAN en la reducción de la prevalencia de la desnutrición crónica fue distinto en las regiones, siendo $3,5 \%$ en Apurímac y $1,5 \%$ en Ayacucho.

Tanto para seguimiento como para evaluación, las encuestas constituyen herramientas de importancia mayor, aunque son claros los beneficios de contar con información de actualización periódica y con una línea de tendencia, debemos reconocer las limitaciones que tiene la información proveniente de encuestas, ello nos ayudará a mejorar la interpretación y la toma de decisiones. La información de encuestas debe ser complementada con aquella que proviene de registros administrativos, estudios específicos y censos. Las encuestas permiten recoger indicadores de resultado; si deseamos identificar en donde se encuentran los problemas para el logro de un resultado, la información podría estar en la entrega de los servicios, el desarrollo de los procesos, la adquisición de los insumos o la programación y ejecución presupuestal requeridas.

Otro aspecto a considerar es que la evidencia científica que respalda cada Programa Presupuestal debe ser revisada y actualizada de manera periódica, ello requerirá 
también de una reestructuración de los indicadores y de su representatividad dentro de las encuestas.

Contribuciones de autoría: JEVH y RRS participaron en todas las etapas de elaboración del artículo, desde la concepción y el diseño del estudio, además de la recolección, el análisis e interpretación de los datos. Ambos autores redactaron el artículo y revisaron los borradores y la versión final, realizando importantes contribuciones.

Fuente de financiamiento: autofinanciado.

Declaración de conflictos de interés: ninguno

\section{REFERENCIAS BIBLIOGRÁFICAS}

1. García López R, García Moreno M. La Gestión para resultados en el desarrollo. Avances y desafíos en América Latina y el Caribe. 2da Edición [Internet]. Washington D. C: Banco Interamericano de Desarrollo; 2010. (citado el 20 de marzo del 2017). Disponible en: https:// goo.gl/wF8MDb

2. Kaufmann J, Sanginés M, García Moreno M. Construyendo gobiernos efectivos. Logros y retos de la gestión pública para resultados en América Latina y el Caribe. 2da Edición. [Internet]. Washington D. C: Banco Interamericano de Desarrollo; 2015. (citado el 20 de marzo del 2017). Disponible en: https://goo.gl/Pjm6Xh

3. Gertler PJ, Martinez S, Premand P, Rawlings LB, Vermeersch CM. La evaluación de impacto en la práctica. 1ra Edición [Internet]. Washington D. C: Banco Mundial; 2011. (citado el 22 de marzo del 2017). Disponible en: http://siteresources.worldbank.org/ INTHDOFFICE/Resources/IEP_ SPANISH_FINAL_110628.pdf

4. Programas Presupuestales [Internet]. Lima: Ministerio de Economía y Finanzas; 2017. (citado el 16 julio 2017). Disponible en: https://www.mef.gob. pe/es/presupuesto-por-resultados/ instrumentos/programas-presupuestales.

5. ENDES 2016 - Informe principal [Internet]. Lima: Instituto Nacional de Estadística e Informática; 2016. (citado el 16 de julio 2017). Disponible en: http:// proyectos.inei.gob.pe/endes/resultados.asp

6. Encuesta Nacional de Hogares sobre Condiciones de Vida y Pobreza 2013 [Internet]. Lima: Instituto Nacional de Estadística e Informática; 2013. (citado el 16 julio de 2017). Disponible en: http:// www.ilo.org/surveydata/index.php/ catalog/358.

7. Encuesta Nacional de Programas Sociales (ENAPRES) 2015 [Internet]. Lima: Instituto Nacional de Estadística e Informática; 2015. (citado el 16 julio de 2017). Disponible en: http://webinei. inei.gob.pe/anda_inei/index.php/ catalog/258.

8. Encuesta a Establecimientos de Salud en la Atención del Control de Crecimiento, Desarrollo y Vacunas en Menores de un año - 2015. [Internet]. Instituto Nacional de Estadística e Informática. (citado el 16 julio de 2017). Disponible en: http:// webinei.inei.gob.pe/anda_inei/index. php/catalog/294.

9. Perú, Características de la Población con Discapacidad. Lima: Instituto Nacional de Estadística e Informática; 2015. (citado el 16 julio de 2017). Disponible en: https://www.inei.gob.pe/media/MenuRecursivo/publicaciones_digitales /Est/ Lib1209/Libro.pdf.

10. Encuestas realizadas por el INEI en el marco del PpR. Lima: Ministerio de Economía y Finanzas; 2015. (citado el 16 Julio de 2017). Disponible en: https:// www.mef.gob.pe/contenidos/presu_ publ/ppr/encuesta_INEI_PPR.pdf

11. Riesgo de Infección por Tuberculosis en Niñas y Niños de 6 y 7 años de edad. Lima: Instituto Nacional de Estadística e Informática; 2013. (citado el 16 julio de 2017). Disponible en: https://www. inei.gob.pe/media/MenuRecursivo/ publicaciones_digitales/Est/Lib1065/ Libro.pdf

12. Presupuesto por Resultados. Lima: Ministerio de Economía y Finanzas; 2008. (citado el 16 julio de 2017). Disponible en: https://www.mef.gob.pe/ contenidos/presu_publ/documentac/ generales/PRESUPUESTO_POR_ RESULTADOS.pdf

13. Ley de Presupuesto del Sector Público para el Año Fiscal 2007. Ley 28927. El Peruano, Normas Legales. pp. 334317. (12 de diciembre de 2006). Disponible en: https://www.mef.gob.pe/contenidos/ presu_publ/sectr_publ/presu_2007/ Ley28927_Presupuesto_2007.pdf.

14. Presupuesto por Resultados. Conceptos y líneas de acción. Documento de Trabajo. Lima: Ministerio de Economía y Finanzas; 2008. (citado el 14 julio de 2017). Disponible en: https://www.mef. gob.pe/contenidos/presu_publ/ppr/ conceptos_lineas_accion.pdf

15. Perú: Evolución de los indicadores de Programas Presupuestales, 2011-2016. Lima: Instituto Nacional de Estadística e Informática; 2017.

16. Resulta, Indicadores de Desempeño de los Programas Presupuestales [Internet]. Lima: Ministerio de Economía y Finanzas. (citado el 16 julio de 2017). Disponible en: http://apps5.mineco.gob. pe/resulta/

17. Microdatos [Internet]. Lima: Instituto Nacional de Estadística e Informática; 2017. (citado el 5 julio de 2017). Disponible en: http://iinei.inei.gob.pe/ microdatos/

18. Perú, Indicadores de los Programas Presupuestales, 2011-2016. Encuesta Demográfica y de Salud Familiar. Lima: Instituto Nacional de Estadística e Informática; 2017.

19. Encuesta Demográfica y de Salud Familiar 2016. Lima: Instituto Nacional de Estadística e Informática; 2017. (citado el 10 julio de 2017). Disponible en: https://www.inei.gob.pe/media/MenuRecursivo/publicaciones_digitales /Est/ Lib1433/index.html

20. Reporte de progreso en la obtención de resultados de los Programas Presupuestales iniciados el 2008 - 2015: Resultados 2015. Lima: Ministerio de Economía y Finanzas; 2016. (citado el 10 julio de 2017). Disponible en: https:// www.mef.gob.pe/contenidos/presu $\mathrm{publ} / \mathrm{ppr} / \mathrm{rpte}$ _progreso2015_cap4.pdf

21. Tercer informe, intervención pública evaluada: servicio de vacunación. Lima: Ministerio de Salud; 2011. (citado el 10 julio de 2017). Disponible en: https:// www.mef.gob.pe/contenidos/presu_ $\mathrm{publ} / \mathrm{ppr} / \mathrm{eval}$ indep/2010_ informe_ final_VACUNACIONES.pdf

22. Intervención: Atención de enfermedades diarreicas agudas (EDA) y las infecciones respiratorias agudas (IRA). En: 
Evaluaciones de Diseño y Ejecución Presupuestal. Herramienta para el logro de resultados en la gestión pública. 1ra edición. Lima: Ministerio de Economía y Finanzas (MEF); 2013. (citado el 10 julio de 2017). Disponible en: https://www. mef.gob.pe/contenidos/presu_publ/ppr/ eval_indep/publicacion_EDEP.pdf

23. Consultoría para la Evaluación del Diseño y Ejecución de presupuestos públicos - Atención del parto normal. Lima: Ministerio de Economía y Finanzas (MEF); 2013. (citado el 10 julio de 2017). Disponible en: https://www.mef. gob.pe/contenidos/presu_publ/ppr/ eval_indep/2013_parto_normal.pdf

24. Castillo Velásquez $\mathrm{O}$, Zúñiga Cárdenas R. Evaluación del Diseño y Ejecución presupuestal de la Intervención Publica “CRED y Municipios Saludables". Lima: Ministerio de Economía y Finanzas (MEF); 2015. (citado el 10 julio de 2017). Disponible en: https://www.mef.gob. pe/contenidos/presu_publ/ppr/eval_ indep/2015_CRED_mun_saludable.pdf

25. Cruzado V. Análisis del impacto del Programa Presupuestal Articulado Nu- tricional sobre la Desnutrición Crónica en niños menores de 5 años. Lima: Dirección General de Presupuesto Público, Ministerio de Economía y Finanzas (MEF); 2012. (citado el 10 julio de 2017). Disponible en: https://www.mef.gob.pe/ contenidos/presu_publ/ estudios/PAN_ final_fusionado.pdf

Correspondencia: José Enrique Velásquez Hurtado Dirección: Av. Paso de los Andes 681. Pueblo Libre, Perú

Teléfono: (+511) 998674127

E-mail:jose.velasquez@upch.pe

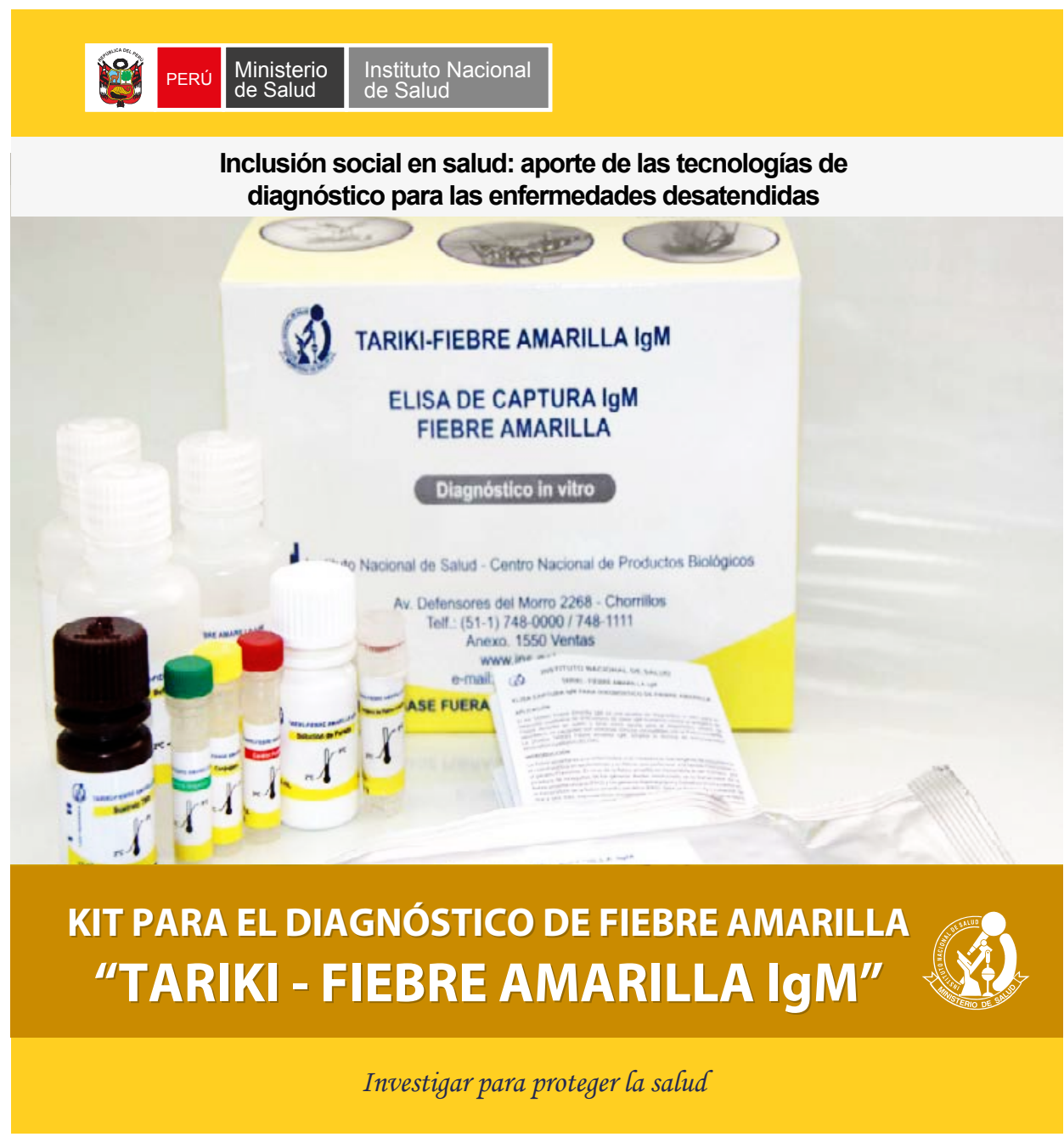

